\title{
Counter-rotating disks in galaxies: dissecting kinematics and stellar populations with 3D spectroscopy
}

\author{
Lodovico Coccato $^{1}$, Lorenzo Morelli ${ }^{2,3}$, Alessandro Pizzella ${ }^{2,3}$, \\ Enrico Maria Corsini ${ }^{2,3}$, Elena Dalla Bontà ${ }^{2,3}$ and \\ Maximilian Fabricius ${ }^{4,5}$ \\ ${ }^{1}$ European Southern Observatory, Karl-Schwarzschild-Straße 2, D-85748, Garching bei \\ München, Germany. \\ ${ }^{2}$ Dipartimento di Fisica ed Astronomia "Galileo Galilei", Università di Padova, vicolo \\ dell'Osservatorio 3, 35122 Padova, Italy. \\ ${ }^{3}$ INAF-Osservatorio Astronomico di Padova, vicolo dell'Osservatorio 5, 35122 Padova, Italy. \\ ${ }^{4}$ Max Planck Institute for Extraterrestrial Physics, Giessenbachstraße, D-85748 Garching, \\ Germany. \\ ${ }^{5}$ University Observatory Munich, Scheinerstra $\beta$ e 1, 81679 Munich, Germany. \\ email: lcoccato@eso.org
}

\begin{abstract}
We present a spectral decomposition technique that separates the contribution of different kinematic components in galaxies from the observed spectrum. This allows to study the kinematics and properties of the stellar populations of the individual components (e.g., bulge, disk, counter-rotating cores, orthogonal structures). Here, we discuss the results of this technique for galaxies that host counter-rotating stellar disks of comparable size. In all the studied cases, the counter-rotating stellar disk is the less massive, the youngest and has different chemical content (metallicity and $\alpha$-elements abundance ratio) than the main galaxy disk. Further applications of the spectral decomposition technique are also discussed.
\end{abstract}

Keywords. galaxies: elliptical and lenticular - galaxies: formation

\section{Introduction}

Galaxies can have a complex kinematic structure, due to the superimposition of multiple components that have different kinematics and stellar populations, such as bulge and disk (in lenticulars and spirals), host spheroid and polar ring (in polar ring galaxies), and counter-rotating stellar disks or kinematically decoupled cored (mainly - but not only in early-type galaxies).

The interesting case of large-scale counter-rotating galaxies, i.e., those that host two stellar components of comparable sizes that rotate along opposite directions, is the topic of this work. The prototype of this class of objects is the E7/S0 galaxy NGC 4550, whose counter-rotating nature was first discovered by Rubin et al. (1992).

\section{Spectral decomposition}

The spectral decomposition technique by Coccato et al. (2011) is an implementation of the penalized pixel fitting code by Cappellari \& Emsellem (2004). For a given spectrum, the code builds two synthetic stellar templates (one for each stellar component) as a linear combination of stellar spectra from an input stellar library. It then convolves these two stellar templates with two Gaussian line-of-sight velocity distributions (LOSVDs), which are characterized by different velocity and velocity dispersion. The input galaxy 


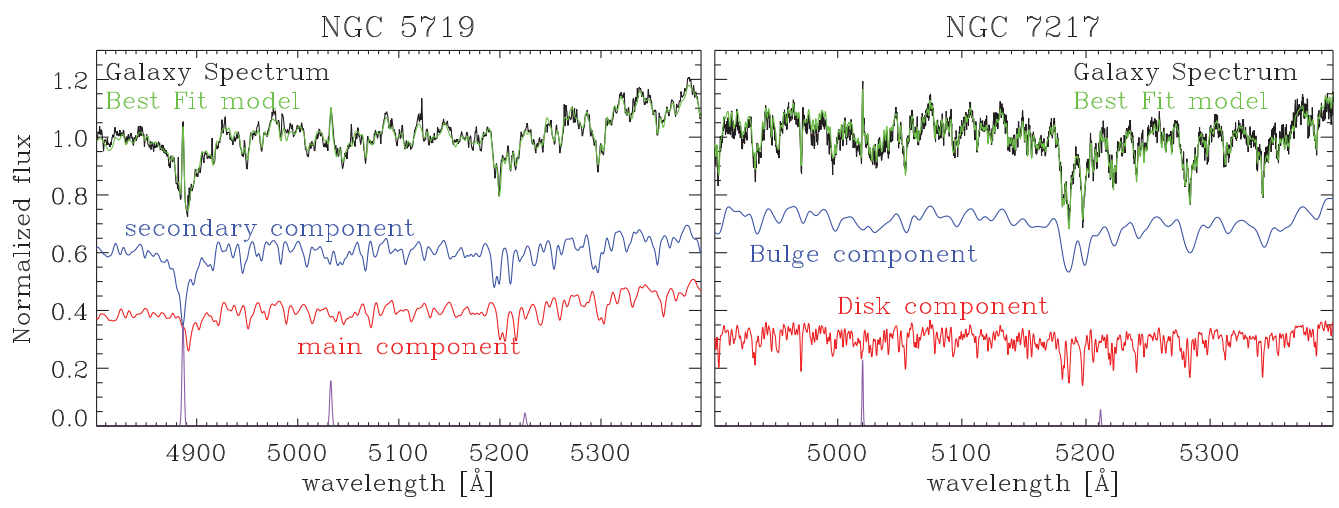

Figure 1. Example of the fit results of the spectral decomposition technique, applied to disentangle the contribution of two counter-rotating stellar disks (left panel, from Coccato et al. 2011), and the contribution of the stars in the bulge and in the disk (right panel, from Fabricius et al. 2014). Black: observed galaxy spectrum; green: best fit model; blue and red: spectra of the two stellar components; purple: gas emission lines.

and stellar spectra are normalized to their continuum level, therefore the contribution of each component to the observed galaxy spectrum is measured in terms of light. Gaussian functions are added to the convolved synthetic templates to account for ionized-gas emission lines. Multiplicative Legendre polynomials are included in the fit to match the shape of the galaxy continuum, and are set to be the same for the two synthetic templates. This accounts for the effects of dust extinction and variations in the instrument transmission. These steps are embedded in a iterative $\chi^{2}$ minimization loop.

The spectral decomposition code returns the spectra of two best-fit synthetic stellar templates and ionized-gas emissions, along with the best-fitting parameters of light contribution, velocity, and velocity dispersion. The line strength of the Lick indices of the two counter-rotating components are then extracted from the two best-fit synthetic templates, and used to infer the properties of the stellar populations of the two components.

Figure 1 shows some examples of results of the spectral decomposition.

\section{Applications and results}

The technique has been successfully applied to disentangle (i) counter-rotating stellar components in galaxies (Coccato et al. 2011, 2013; Pizzella et al. 2014), (ii) the kinematics of stars in bulge and disk (Fabricius et al. 2014), and (iii) the kinematics of the host galaxy and orthogonal disk in polar disk galaxies (Coccato et al. 2014).

Here, we describe the results obtained for a sample of galaxies that host counterrotating stellar disks of comparable size: NGC 3959, NGC 4183, NGC 4550, and NGC 5719. Observations were obtained with the VIMOS integral field unit at the VLT (Chile), except for NGC 4138, which was observed with the B\&C spectrograph at the 1.22-m telescope at Padova University (Italy). In all the studied galaxies, we detect the presence of an extended secondary stellar component, which is counter-rotating with respect the more luminous and massive stellar component. In addition, the secondary component is rotating along the same direction as the ionized gas. The measurements of the equivalent width of the absorption line features $(\mathrm{H} \beta, \mathrm{Mgb}, \mathrm{Fe} 5270$, and Fe5335) reveal that the secondary stellar component is always younger than the main stellar component, and it is more metal poor. Such a difference in stellar population is particularly pronounced in NGC 5719 (Figure 2). 

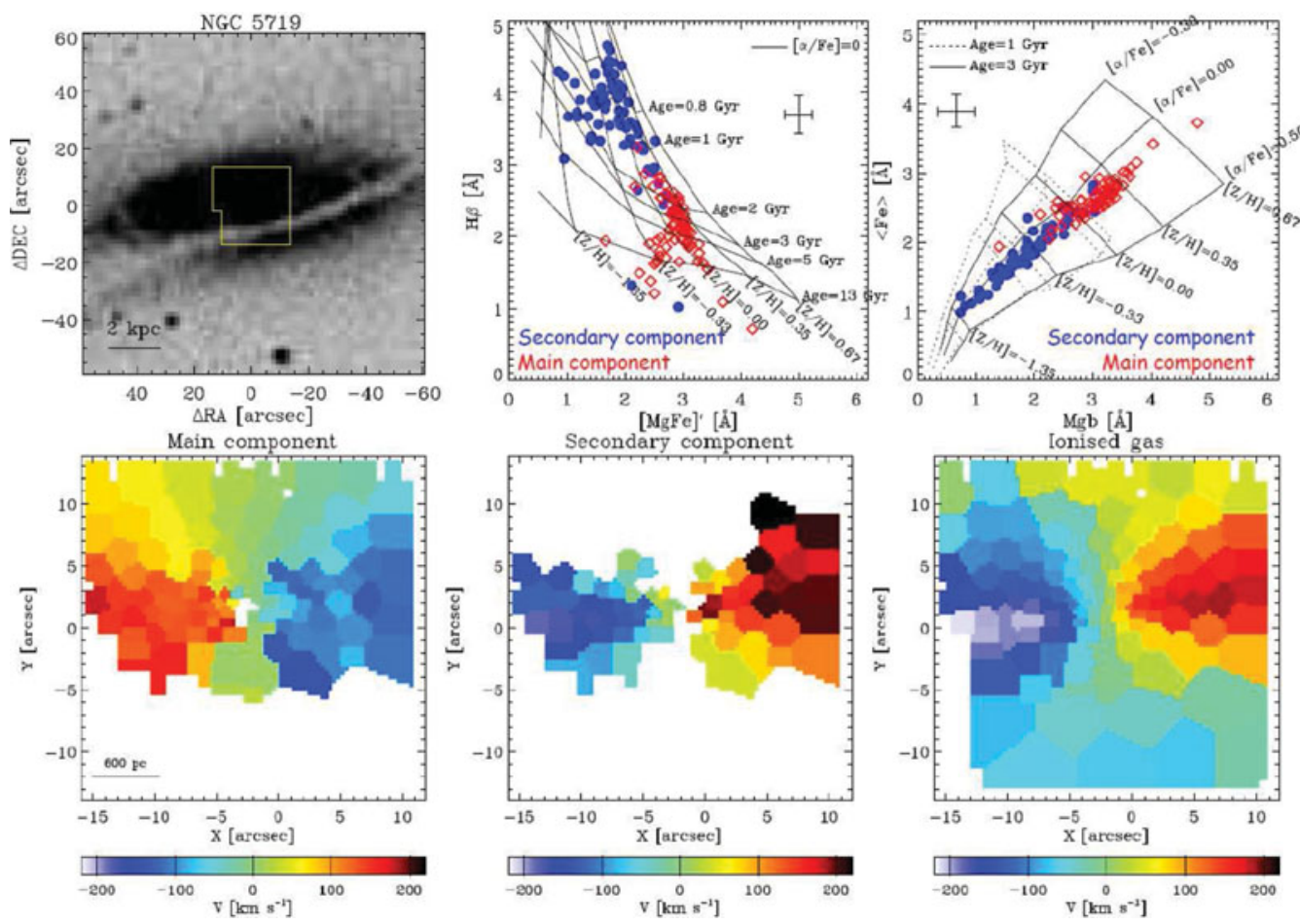

Figure 2. Top left panel: image of the Sa galaxy NGC 5719, the yellow region indicates the footprint of the VIMOS/IFU field of view. Top central and right panels: line strengths of the Lick indices measured on the main (red) and secondary (blue) stellar components: $\mathrm{H} \beta$, combined magnesium-iron index [MgFe]' (Gorgas et al. 1990), Mgb, and average iron index $<\mathrm{Fe}>$ (Thomas et al. 2003). The black lines show the prediction of single stellar population models (Thomas et al. 2011). The bottom panels show the measured two-dimensional velocity fields of the main (left) and secondary (central) stellar components, and the ionized gas component (right). Adapted from Coccato et al. (2011).

In the case of IFU observations, the spectral decomposition allows also to investigate the morphology of the decoupled structures by comparing their light contribution to the reconstructed image of the galaxy. It is therefore possible to unveil the real extension and geometrical properties such as orientation, ellipticity, and scale length of the decoupled disks. In Figure 3 we compare the reconstructed images and the surface brightness radial profiles of the counter-rotating disks observed in NGC 3593. The secondary components overshines the main stellar disk within $\sim 10^{\prime \prime}$. A single-component fit would reveal the presence of the secondary component only in the inner regions, by revealing the kinematic signature of a kinematically decoupled core (KDC, e.g. Bender 1988).

The combination of the luminosity profile and stellar mass-to-light ratio, as inferred from the stellar population analysis, shows that the secondary component has a lower mass density radial profile than the main component, also in the inner regions where it dominates in light.

\section{Conclusions}

The stellar population properties and kinematics of the counter-rotating stellar disks are consistent with the formation scenario where a pre-existing galaxy acquired external gas on retrograde orbits from a companion galaxy or the external medium (e.g. Thakar \& Ryden 1996; Pizzella et al. 2004). Depending on the amount of acquired material, the 

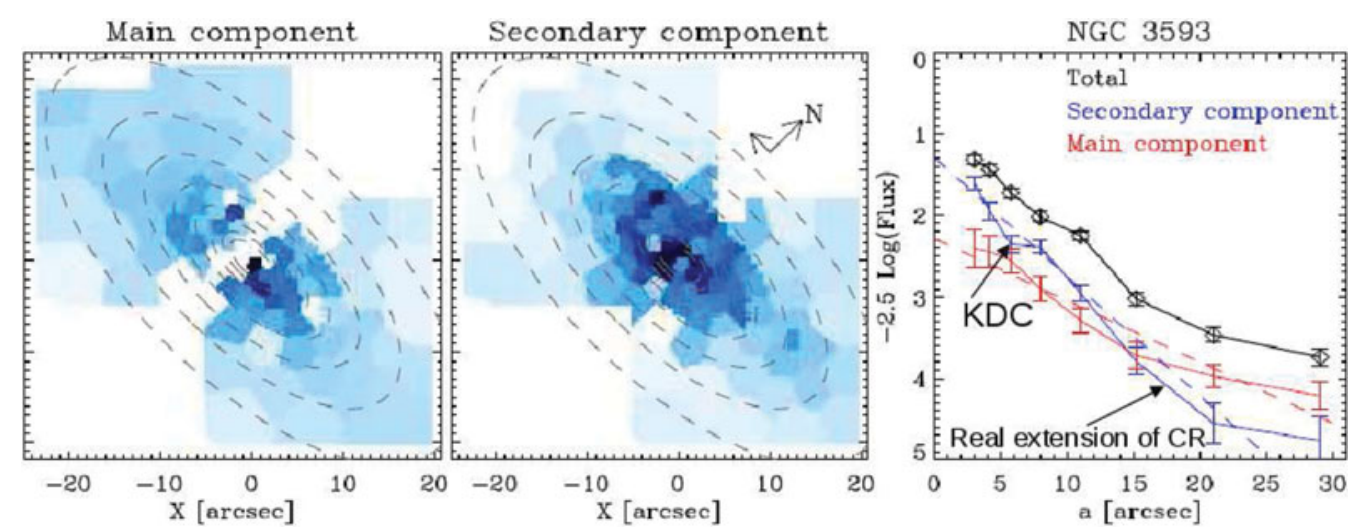

Figure 3. Maps (left and central panels) and radial profiles (right panel) of the surface brightness of the main and the secondary stellar components of NGC 3593. The black, red, and blue solid lines correspond to the total surface brightness and to the surface brightness of the main and the secondary stellar components, respectively. The dashed red and dashed blue lines correspond to the best-fitting exponential disks to the surface brightness of the main and secondary stellar components, respectively. Dashed ellipses represent the boundaries of the elliptical annuli where the median surface brightnesses were computed. Adapted from Coccato et al. (2013).

new gas removes the pre-existing gas and sets onto a disk in retrograde rotation. The new gas forms thevv stars of the counter-rotating secondary component. In the case of NGC 5719 we have a direct of the on-going acquisition process (Vergani et al. 2007).

Despite of their discovery more than 30 years ago, counter-rotating galaxies still represent a challenging subject (see Bertola \& Corsini 1999; Corsini 2014 for reviews). We have showed that the spectral decomposition allows to measure the properties of the stellar populations of both components, and to probe the predictions of the different formation scenarios. Forthcoming large integral field spectroscopic surveys such as MANGA (Bundy et al. 2014), will allow to increase the statistics on these objects, providing fundamental clues to constrain their formation scenarios.

\section{References}

Bender, R. 1988, A\&\&A, 202, L5

Bertola, F. \& Corsini, E. M. 1999, in IAU Symposium, Vol. 186, Galaxy Interactions at Low and High Redshift, ed. J. E. Barnes \& D. B. Sanders, 149

Bundy, K., Bershady, M. A., Law, D., et al. 2014, ApJ submitted

Cappellari, M. \& Emsellem, E. 2004, PASP, 116, 138

Coccato, L., Iodice, E., \& Arnaboldi, M. 2014, A\& $A$ in press (ArXiv:1409.1235)

Coccato, L., Morelli, L., Corsini, E. M., et al. 2011, MNRAS, 412, L113

Coccato, L., Morelli, L., Pizzella, A., et al. 2013, A\&A, 549, A3

Corsini, E. M. 2014, in Astronomical Society of the Pacific Conference Series, Vol. 486, Astronomical Society of the Pacific Conference Series, ed. E. Iodice \& E. M. Corsini, 51

Fabricius, M. H., Coccato, L., Bender, R., et al. 2014, MNRAS, 441, 2212

Gorgas, J., Efstathiou, G., \& Aragon Salamanca, A. 1990, MNRAS, 245, 217

Pizzella, A., Corsini, E. M., Vega Beltrán, J. C., \& Bertola, F. 2004, A\& 4 A, 424, 447

Pizzella, A., Morelli, L., Corsini, E. M., et al. 2014, A\&A in press (ArXiv:1409.3086)

Rubin, V. C., Graham, J. A., \& Kenney, J. D. P. 1992, ApJ, 394, L9

Thakar, A. R. \& Ryden, B. S. 1996, ApJ, 461, 55

Thomas, D., Maraston, C., \& Bender, R. 2003, MNRAS, 339, 897

Thomas, D., Maraston, C., \& Johansson, J. 2011, MNRAS, 412, 2183

Vergani, D., Pizzella, A., Corsini, et al. 2007, A\& A, 463, 883 